\title{
Nuclear data sensitivity and uncertainty assessment of sodium voiding reactivity coefficients of an ASTRID-like sodium fast reactor
}

\author{
García-Herranz Nuria ${ }^{1, \text { a }}$, Panadero Anne-Laurène ${ }^{2}$, Martinez Ana ${ }^{1}$, Pelloni Sandro² ${ }^{2}$ Mikityuk Konstantin $^{2}$, \\ and Pautz Andreas ${ }^{2}$ \\ 1 UPM, Universidad Politécnica de Madrid, Jose Gutierrez Abascal, 2, 28006 Madrid, Spain \\ 2 FAST project, Laboratory for Reactor Physics and Systems Behaviour, Paul Scherrer Institut, 5232 Villigen, Switzerland
}

\begin{abstract}
The EU 7th Framework ESNII+ project was launched in 2013 with the strategic orientation of preparing ESNII for Horizon 2020. ESNII stands for the European Industrial Initiative on Nuclear Energy, created by the European Commission in 2010 to promote the development of a new generation of nuclear systems in order to provide a sustainable solution to cope with Europe's growing energy needs while meeting the greenhouse gas emissions reduction target. The designs selected by the ESNII+ project are technological demonstrators of Generation-IV systems. The prototype for the sodium cooled fast reactor technology is ASTRID (standing for Advanced Sodium Technological Reactor for Industrial Demonstration), which detailed design phase is foreseen to be initiated in 2019.

The ASTRID core has a peculiar design which was created in order to tackle the main neutronic challenge of sodium cooled fast reactors: the inherent overall positive reactivity feedback in case of sodium voiding occurring in the core. Indeed, the core is claimed by its designers to have an overall negative reactivity feedback in this scenario. This feature was demonstrated for an ASTRID-like core within the ESNII+ framework studies performed by nine European institutions. In order to shift the paradigm towards bestestimate plus uncertainties, the nuclear data sensitivity analysis and uncertainty propagation on reactivity coefficients has to be carried out.

The goal of this work is to assess the impact of nuclear data uncertainties on sodium voiding reactivity feedback coefficients in order to get a more complete picture of the actual safety margins of the ASTRID low void-core design. The nuclear data sensitivity analysis is performed in parallel using SCALE TSUNAMI3D and the newly developed GPT SERPENT 2 module. A comparison is carried out between the two methodologies. Uncertainty on the sodium reactivity feedbacks is then calculated using TSAR module of SCALE and the necessary safety margins conclusions are drawn.
\end{abstract}

\section{Introduction}

The European Industrial Initiative on Nuclear Energy (ESNII) promotes the development and demonstration of advanced fast neutron Gen IV reactor technologies under the umbrella of the European Platform for Sustainable Nuclear Energy (SNETP). In order to prepare ESNII for Horizon 2020, the EURATOM 7th Framework Project ESNII+ was launched in 2013 [1]. It aims to establish the roadmap for the development of fast spectrum reactors.

The prototype for the sodium cooled fast reactor technology is ASTRID (standing for Advanced Sodium Technological Reactor for Industrial Demonstration), which detailed design phase is foreseen to be initiated in 2019. ASTRID will allow to demonstrate the capability to master the mature sodium technology with improved safety characteristics, integrating operational feedback of past sodium reactors while increasing the robustness of this technology. A dedicated ASTRID project [2], led by CEA, deals with the main research and technological development of the prototype.

\footnotetext{
a e-mail: nuria.garcia.herranz@upm.es
}

The ASTRID core has a peculiar design which was created in order to tackle the main neutronics challenge of sodium cooled fast reactors: the inherent overall positive reactivity feedback in case of sodium void, which may result from boiling, a leak of the sodium circuit or due to gas going through the core. Indeed, the core is claimed by its designers to have an overall negative reactivity feedback in this scenario [3], which would prevent and mitigate severe accidents. The confirmation of that behaviour is one of the main areas for research concerning ASTRID. This feature was demonstrated for an ASTRID-like core at EndOf-Cycle (EoC) conditions within the ESNII+ Project. Studies performed by nine institutions [4] using different state-of-the-art deterministic and Monte Carlo neutronics codes and data libraries confirmed that the total voiding has a negative effect (around $-400 \mathrm{pcm}$ average), resulting from a positive effect when voiding fuel regions and a negative effect when voiding plenum regions. However while a reasonable agreement was found in predictions of the void effect in the fuel regions, larger discrepancies were observed in the sodium plenum region (where the angular flux becomes very anisotropic), reflecting the 


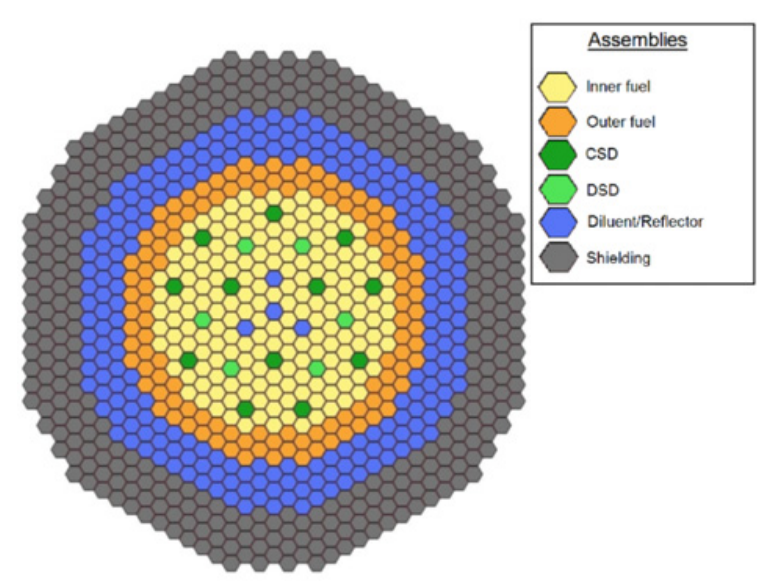

Figure 1. Cross-sectional view of the ASTRID-like core (subassembly pitch of $17.4 \mathrm{~cm}$ at $20^{\circ} \mathrm{C}$ ) [7].

complexity of the neutron transport modelling in those zones.

The uncertainty quantification becomes then necessary in order to get a more complete picture of the actual safety margins of this ASTRID-like innovative core design. Uncertainties due to nuclear data and calculation scheme should be estimated and compared to experimental results (past available experiments or ad-hoc designed experiments).

The goal of this work is to assess the impact of nuclear data uncertainties on the spatial-dependent sodium voiding reactivity coefficients. Two methodologies are applied to compute the sensitivity coefficients: the multigroup TSUNAMI-3D module from SCALE6.1 system [5] and the GPT module from the Monte Carlo code SERPENT2 [6] employing in both cases the ENDF/B-VII.0 data library. Then, uncertainties are propagated using TSAR (SCALE Tool for Sensitivity Analysis of Reactivity) and SCALE6 covariance matrices ${ }^{1}$, so that computed uncertainties could be compared with the target ones required for detailed design. The list with the main contributors to the uncertainties in sodium void is also obtained and compared to the dependencies of the multiplication factor at nominal conditions.

\section{ASTRID-like core configuration}

The sodium-cooled core configuration assumed for this study consists of a highly axially and radially heterogeneous design of $1500 \mathrm{MW}$ of thermal power. The core was modelled at EoC and nominal operating conditions (specified in [4]). As depicted in Fig. 1, the core is composed of 291 hexagonal sub-assemblies (with a triangular arrangement of 217 pins). Two MOX-fuelled fissile zones, inner and outer can be observed, containing the inner fuel a Pu mass fraction of $\sim 19 \%$ at EoC and the outer fuel $\sim 17 \%$. Three rings of radial reflector assemblies and four rings of radial shielding assemblies surround the active core. The axial layout in Fig. 2 shows an absorbing shielding on the top (aimed to prevent neutrons from going back to the core during sodium voiding), a large sodium plenum at the top of the active core (aimed at maximizing

${ }^{1}$ SCALE6 matrices are among the most complete compilation of covariances and, although not only based on covariances of evaluated libraries, are useful to estimate the global uncertainties.

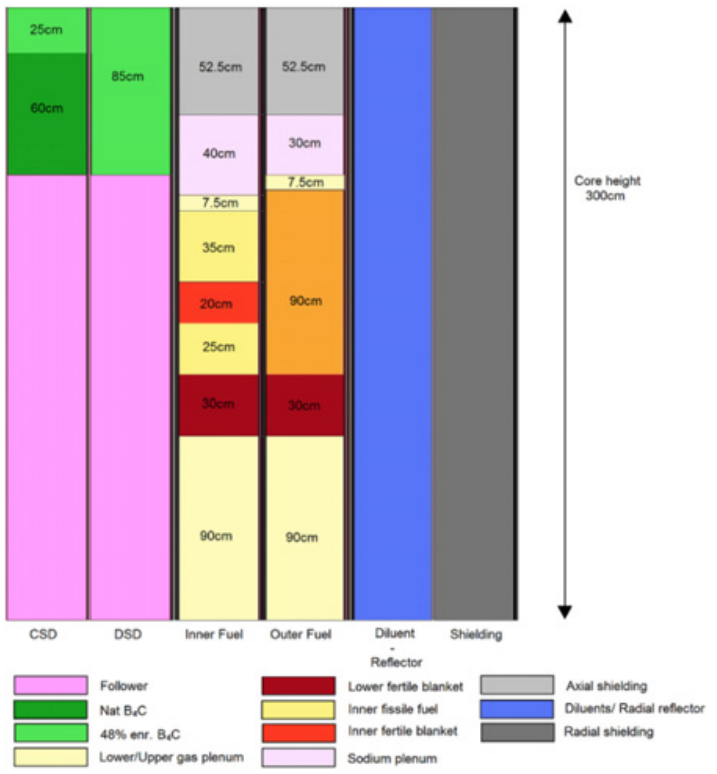

Figure 2. Axial view of the ASTRID-like core assemblies (heights at $20^{\circ} \mathrm{C}$ ) [7].

neutron leakage) and a thick fertile blanket below the active core (aimed to increase the flux level at the upper surface especially in case of sodium voiding). Different heights for inner and outer fissile fuel regions are foreseen, with an internal axial blanket in the inner region. It can be noticed that the resulting active core is rather flat, with a reduced core height $(\sim 0.9 \mathrm{~m}$ for the outer zone) compared to the radial width ( $\sim 3.3 \mathrm{~m}$ of fissile core diameter).

\section{Sensitivity methodologies applied to the ASTRID-like core}

The main advantages and critical aspects of TSUNAMI3D and SERPENT sensitivity techniques for application to an ASTRID-like core are pointed out.

\subsection{TSUNAMI-3D sequence}

Multigroup (MG) TSUNAMI-3D is a SCALE module that provides complete eigenvalue sensitivities to nuclear data (sum of explicit and implicit components). Explicit sensitivity coefficients are computed from the product of forward and adjoint fluxes (provided by the MG Monte Carlo KENO code) via first order perturbation theory. Implicit components account for the changes in MG cross-sections via self-shielding perturbations. MG TSUNAMI-3D has one main advantage with respect to other methodologies: all sensitivity coefficients for all reactions are efficiently calculated since the forward and adjoint fluxes are tallied separately. However, a critical aspect of this approach is that the relevant energy, spatial and directional dependences of the fluxes must be captured in order to obtain accurate sensitivity coefficients (discrepancies by less than $5 \%$ with respect to reference sensitivities obtained by Direct Perturbation).

For this study, concerning energy dependence, the ENDF/B-VII.0 238 group cross section library (optimized for thermal system applications) was used since no optimal multigroup library for fast systems is available in SCALE so far. Concerning directional and spatial resolutions, an 
analysis of the TSUNAMI-3D parameters that control the calculation of the angular fluxes was performed for the ASTRID-like core in order to find the optimal set of parameters yielding accurate results together with reasonable computing time and memory requirements [8]. It was found that a third-order of the spherical harmonics expansion of the angular flux was adequate to catch the anisotropies of the flux if a refined enough spatial flux mesh is set. At least a mesh of $10 \mathrm{~cm}$ for all material regions of the core model was required, except for the sodium upper plenum, which required a $5 \mathrm{~cm}$ mesh resolution, being the most sensitive material to the discretization.

\subsection{SERPENT 2 GPT module}

SERPENT 2 is a Monte Carlo code currently being developed at VTT Technical Research Center of Finland [9]. This Monte Carlo code is attractive for this study for its following features: the continuous energy calculation scheme and the Woodcock delta tracking method which decreases the calculation time in fast spectrum reactor calculations; and the possibility to use the GPT module to calculate sensitivity coefficients arising from cross-section perturbation.

SERPENT 2 GPT module was developed in 2015 and uses a collision-based approach to sensitivityperturbation calculations [6]. The cross-section perturbation is simulated by biasing the sampling process through the modification of the collision probability function, in addition to the introduction of a collision acceptance/rejection algorithm which follows the inverse bias of the probability density function, allowing the physics of the system to remain unchanged. The score of acceptance/rejection events is stored in the neutron histories, being possible to calculate the sensitivity coefficient of each cross-section perturbation from that score.

The SERPENT 2 GPT module has currently the capabilities of simulating the perturbation of the following spectral parameters: fission, $(n, \gamma),(n, n),\left(n, n^{\prime}\right)$, nubar (delayed, prompt and total), (n,xn). The neutron spectrum $\chi$ (delayed, prompt and total), already implemented in the module, was not available at the time of this work.

The sensitivity coefficients are calculated for each isotope and spatial zone of material considered. Given the collision based approach of the methodology, the computational time of the simulation is strongly correlated to the number of isotopes considered (running time of each cycle of the simulation), the number of energy groups considered as well as the number of spatial zones (need for higher statistics to obtain reasonable convergence in the energy groups/spatial zones). Thus, the SERPENT 2 GPT module is fast running for a small number of isotopes, energy groups and spatial zones, and becomes too computationally expensive in other cases. This implies the principal constraint to the system: in order to calculate sensitivity coefficients, the isotopes have to be defined prior to the simulations.

For the moment, the SERPENT 2 GPT module has no capabilities of propagating the sensitivity coefficients to $\mathrm{k}$-eff using covariance data, therefore, in order to assess the impact on k-eff and reactivity, the sensitivity coefficients are implemented in TSAR module of SCALE. As a

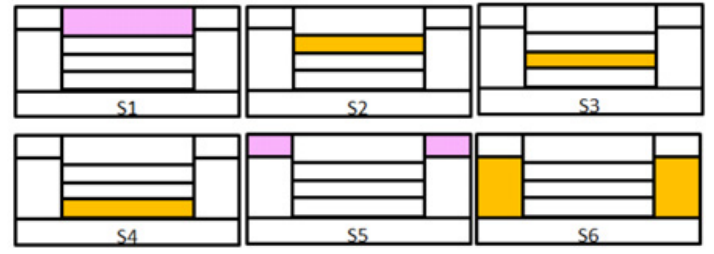

Figure 3. Schematic representation of the 6 voiding scenarios [4]. Colored zones represent the voided zones, with pink illustrating sodium plenum and orange fuel zone.

consequence, the energy grid was chosen as corresponding to the 44 groups TSUNAMI grid, for its reasonable number of bins and its compatibility to TSAR.

\section{S/U analysis}

The sodium void reactivity effect can be expressed as a variation of the reactivity of the unperturbed or nominal state (characterized by a multiplication factor $k$ ) and the perturbed or sodium voided state (characterized by a multiplication factor $k_{p}$ ):

$$
\Delta \rho=\left(1-\frac{1}{k_{\mathrm{p}}}\right)-\left(1-\frac{1}{k}\right)=\frac{k_{\mathrm{p}}-k}{k_{\mathrm{p}} \cdot k}
$$

Let us denote $S_{k, \alpha}$ and $S_{k_{\mathrm{p}}, \alpha}$ the sensitivity coefficients of the nominal and perturbed multiplication factors to the parameter $\alpha$ (corresponding here to a pair nuclidereaction):

$$
S_{k, \alpha}=\frac{\delta k / k}{\delta \alpha / \alpha} \quad S_{k_{\mathrm{p}}, \alpha}=\frac{\delta k_{\mathrm{p}} / k_{\mathrm{p}}}{\delta \alpha / \alpha}
$$

TSAR module combines those sensitivities to compute the relative sensitivity coefficient (at first-order) for $\Delta \rho$ to variations of parameter $\alpha$ :

$$
S_{\Delta \rho, \alpha}=\frac{\delta(\Delta \rho) /|\Delta \rho|}{\delta \alpha / \alpha}=\frac{\frac{S_{\mathrm{kp} \alpha}}{k_{\mathrm{p}}}-\frac{S_{k, \alpha}}{k}}{|\Delta \rho|}
$$

The uncertainty $\varepsilon_{\mathrm{x}}$ on the multiplication factor $(x=k)$ or on the reactivity variation $(x=\Delta \rho)$ due to nuclear data is then calculated by TSAR using the sensitivity matrix $S_{x, \alpha}$ and the relative covariance matrix $\mathrm{COV}_{\alpha \alpha}$ (describing nuclear data uncertainties and correlations) with the sandwich formula:

$$
\varepsilon_{x}=\frac{\Delta x}{x}=\sqrt{\mathbf{S}_{\mathbf{x}, \boldsymbol{\alpha}}^{\mathbf{T}} \operatorname{COV}_{\boldsymbol{\alpha} \alpha} \mathbf{S}_{\mathbf{x}, \boldsymbol{\alpha}}}
$$

In the present work, two covariance matrices are applied: the 44-group SCALE6.1 covariance matrix and the recently released 56-group SCALE6.2 covariance matrix, which include data from ENDF/B-VII.1 covariance evaluations, retaining also data from SCALE6.1.

The following core configurations are considered:

- The nominal core at nominal operating conditions described in [4].

- Six perturbed states, described in the ESNII+ project [4], each of them representing a scenario defined by the voiding of the coolant in a region of the core (see Fig. 3 and Table 1). 
Table 1. Description of the voiding scenarios (red cells correspond to sodium voided inside the wrapper, green cells to sodium at nominal conditions) [7].

\begin{tabular}{|c|l|c|c|c|c|c|c|}
\hline \multicolumn{2}{|c|}{ Voiding scenarios parameters } & \multicolumn{5}{|c|}{ Scenario number } \\
\hline Assemblies & Assembly region & $\mathbf{1}$ & $\mathbf{2}$ & $\mathbf{3}$ & $\mathbf{4}$ & $\mathbf{5}$ & $\mathbf{6}$ \\
\hline \multirow{4}{*}{ Inner fuel } & Axial shielding & & & & & & \\
\hline & Sodium plenum & & & & & & \\
\hline & Upper gas plenum & & & & & & \\
\hline & Upper fissile fuel & & & & & & \\
\hline & Inner fertile blanket & & & & & & \\
\hline & Lower fissile fuel & & & & & & \\
\hline \multirow{4}{*}{ Outer fuel } & Axial shielding & & & & & & \\
\hline & Sodium plenum & & & & & & \\
\hline & Upper gas plenum & & & & & & \\
\hline & Fissile fuel & & & & & & \\
\hline
\end{tabular}

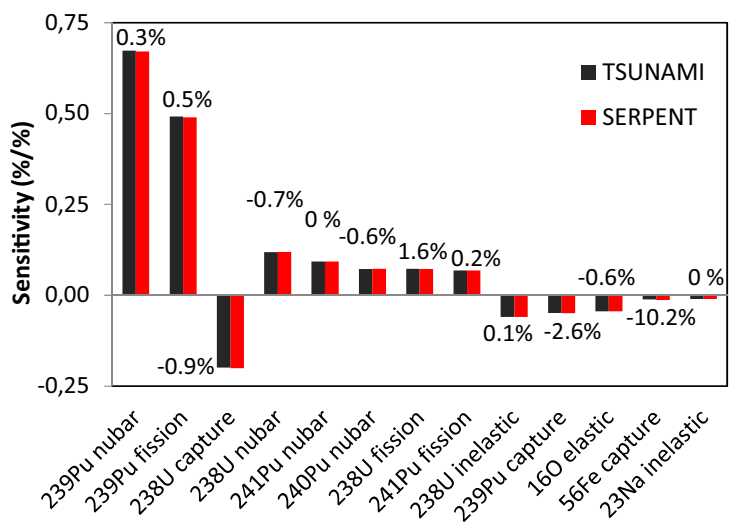

Figure 4. ISC of sodium void reactivity for scenario S1. Relative differences SERPENT-SCALE on the top of the bars.

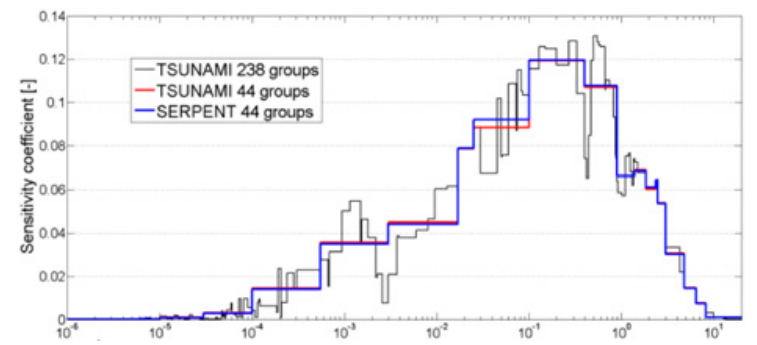

Figure 5. Sensitivity profile of ${ }^{239} \mathrm{Pu}$ nubar for the nominal keff provided by TSUNAMI (in 238 and 44 energy groups) and SERPENT (in 44 energy groups).

\section{1. $\mathrm{S} / \mathrm{U}$ in k-eff at nominal conditions}

A sensitivity analysis of the k-eff multiplication constant at nominal conditions was performed. The obtained Integrated Sensitivity Coefficients (ISC) are in Fig. 4 for a list of selected nuclide-reaction pairs. This selection corresponds to the 10 reactions with the highest sensitivities together with the 3 reactions for ${ }^{16} \mathrm{O},{ }^{56} \mathrm{Fe}$ and ${ }^{23} \mathrm{Na}$ that contribute the most to the k-eff uncertainty.

A very good agreement in the ISC for the whole core is observed, with differences lower than $3 \%$ for the selected reactions except for Fe-56 capture which exhibits discrepancies of $\sim 10 \%$. Energy-dependent sensitivity profiles for those quantities were also compared, being the profiles very close for fuel isotopes (see the profile for ${ }^{239} \mathrm{Pu}$ nubar in Fig. 5).
Table 2. Uncertainties in nominal k-eff (relative values in $\mathrm{pcm}$ ) obtained with TSUNAMI and SERPENT sensitivities based on different covariance libraries and different number of reactions.

\begin{tabular}{|c|c|c|c|}
\hline \multirow{2}{*}{ Case } & Cross-section- & \multicolumn{2}{|c|}{$\Delta \mathrm{k} / \mathrm{k}(\mathrm{pcm})$} \\
\cline { 3 - 4 } & covariance data file & SCALE & SERPENT \\
\hline 13 reactions & 44groupcov & 1185 & 1182 \\
\hline $\begin{array}{c}\text { All reactions } \\
\text { for selected isotopes }\end{array}$ & 44groupcov & & 1280 \\
\hline All isotopes & 56 groupcov7.1 & & 1078 \\
\cline { 3 - 4 } and reactions & 44groupcov & 1318 & \\
\cline { 2 - 4 } & 56 groupcov7.1 & 1131 & \\
\hline
\end{tabular}

The uncertainties in k-eff are shown in Table 2 . Concerning the use of different covariance data, it is noted that the use of SCALE6.2 covariance matrix rather than SCALE6.1 reduces the overall k-eff uncertainty in $\sim 200 \mathrm{pcm}$, mainly due to the reduction of nuclear data uncertainties for ${ }^{239} \mathrm{Pu} v$ and $\chi,{ }^{240} \mathrm{Pu} v$ and ${ }^{238} \mathrm{Pu}$ fission.

Concerning the different codes and number of reactions, it is shown that if only the sensitivity coefficients for the 13 selected nuclide-reaction pairs are used, k-eff uncertainties calculated with TSUNAMI and SERPENT are almost identical (1185 and $1182 \mathrm{pcm}$ respectively). If all reactions for the selected nuclides are considered in SERPENT, the total uncertainty approaches the SCALE predicted value (which takes into account all reactions for all isotopes), being the discrepancy mainly due to the important contribution of ${ }^{239} \mathrm{Pu} \chi$, which was not considered in SERPENT.

The uncertainty breakdown contributions to the overall uncertainty in k-eff based on SCALE6.1 covariance matrix are listed in Table 3, second column, being the $\mathrm{U}$ and $\mathrm{Pu}$ cross-section uncertainties the major contributors. The contributions estimated with both codes show small differences, as expected due to the use of the same covariance data with very similar sensitivity coefficients.

\section{2. $S / U$ in sodium void effects}

A sensitivity analysis of the spatial-dependent sodium void effect allows identifying the key quantities that can lead to biases in the reactivity response. In Fig. 6, the ISC of the highest sensitivities for scenario 1 (corresponding to the inner sodium plenum voiding) are represented. The largest discrepancies between SCALE and SERPENT are for ${ }^{238} \mathrm{U}$ capture and elastic cross-sections. The same key reactions were found for scenario 5 (corresponding to the outer sodium plenum voiding). That indicates that an accurate modelling of the anisotropic elastic scattering of ${ }^{23} \mathrm{Na},{ }^{56} \mathrm{Fe}$ and ${ }^{16} \mathrm{O}$ is a key point to get accurate estimations of the sodium void at plenum regions, being inconsequential the inelastic scattering effects.

Table 4 shows the void reactivity worth for the six scenarios and total uncertainties estimated with both covariance matrices. SCALE results agree quite well with the values predicted by SERPENT. Using the 56group SCALE6. 2 covariance matrix instead of SCALE6.1 increases slightly sodium void uncertainties, unlike k-eff uncertainties (see Table 2).

The break-down by isotope and reaction are provided in Table 3 (columns 3-8) only for the application of SCALE6.1 covariance library. Overall uncertainties given 
Table 3. Major nuclide/reactions contributing up to $95 \%$ to the overall relative uncertainty in nominal k-eff and sodium void effects using SCALE6.1 covariance data.

\begin{tabular}{|c|c|c|c|c|c|c|c|c|c|c|c|c|c|c|}
\hline \multirow{3}{*}{ Quantity } & \multirow{2}{*}{\multicolumn{2}{|c|}{$\begin{array}{c}\Delta k / k(\%) \\
\text { NOMINAL }\end{array}$}} & \multicolumn{12}{|c|}{$\varepsilon_{\Delta \rho}(\%)$} \\
\hline & & & \multicolumn{2}{|c|}{ S1 } & \multicolumn{2}{|c|}{ S2 } & \multicolumn{2}{|c|}{ S3 } & \multicolumn{2}{|c|}{ S4 } & \multicolumn{2}{|c|}{ S5 } & \multicolumn{2}{|c|}{ S6 } \\
\hline & $\begin{array}{c}\text { SCA } \\
\text { LE }\end{array}$ & $\begin{array}{c}\text { SER } \\
\text { PENT }\end{array}$ & $\begin{array}{c}\text { SCA } \\
\text { LE }\end{array}$ & $\begin{array}{c}\text { SER } \\
\text { PENT }\end{array}$ & $\begin{array}{c}\text { SCA } \\
\text { LE }\end{array}$ & $\begin{array}{c}\text { SER } \\
\text { PENT }\end{array}$ & $\begin{array}{c}\text { SCA } \\
\text { LE }\end{array}$ & $\begin{array}{c}\text { SER } \\
\text { PENT }\end{array}$ & $\begin{array}{c}\text { SCA } \\
\text { LE }\end{array}$ & $\begin{array}{c}\text { SER } \\
\text { PENT }\end{array}$ & $\begin{array}{c}\text { SCA } \\
\text { LE }\end{array}$ & $\begin{array}{c}\text { SER } \\
\text { PENT }\end{array}$ & $\begin{array}{c}\text { SCA } \\
\text { LE }\end{array}$ & $\begin{array}{c}\text { SER } \\
\text { PENT }\end{array}$ \\
\hline${ }^{238} \mathrm{U}$ elastic & & & & & & & & & & & & 0.93 & & \\
\hline${ }^{238} \mathrm{U}$ inelastic & 0.92 & 0.91 & 2.14 & 1.90 & 2.75 & 2.32 & 2.70 & 3.26 & 4.30 & 3.65 & 2.18 & 1.86 & 5.36 & 6.76 \\
\hline${ }^{238} \mathrm{U}_{\text {elastic-inelastic }}{ }^{b}$ & 0.21 & 0.21 & 0.84 & 0.37 & & -1.26 & & & 1.24 & -1.78 & 0.83 & -1.30 & 2.58 & \\
\hline${ }^{238} \mathrm{U}$ capture & 0.27 & 0.27 & & & 1.92 & 1.56 & 1.46 & 1.20 & 1.37 & 1.26 & 0.36 & & 3.37 & 3.27 \\
\hline${ }^{238} \mathrm{U}$ nubar & & & 0.37 & & & & & & & & & 0.35 & & \\
\hline $\begin{array}{l}{ }^{238} \mathrm{Pu} \text { fission } \\
{ }^{239} \mathrm{Pu} \text { elastic }\end{array}$ & & & & & & & & & & & & 0.36 & 2.09 & \\
\hline${ }^{239} \mathrm{Pu}$ elastic-inelastic & & & & & & & & & & & & 0.44 & & \\
\hline${ }^{239} \mathrm{Pu}$ capture & 0.25 & 0.25 & & & 1.23 & 1.01 & & & & & & & 2.12 & \\
\hline${ }^{239} \mathrm{Pu}$ fission & & & & & 1.24 & 1.10 & 0.95 & & 1.21 & 1.11 & & 0.37 & & 2.21 \\
\hline${ }^{239} \mathrm{Pu}$ nubar & 0.66 & 0.67 & 0.79 & 0.81 & 2.32 & 2.09 & 1.36 & 1.22 & 1.96 & 1.85 & 0.80 & 0.86 & 3.95 & 4.21 \\
\hline${ }^{239} \mathrm{Pu} \chi$ & 0.29 & a & 0.65 & a & & & & & & & 0.52 & & & \\
\hline${ }^{240} \mathrm{Pu}$ nubar & 0.21 & 0.21 & & & & & & & & & & & & \\
\hline${ }^{16} \mathrm{O}$ elastic & & & & & & & 0.98 & 1.18 & & & & 0.47 & & \\
\hline${ }^{23} \mathrm{Na}$ elastic & & & 1.30 & 1.21 & 2.67 & 2.30 & 2.28 & 1.98 & 2.22 & 2.33 & 1.33 & 1.39 & 3.35 & 3.20 \\
\hline${ }^{23} \mathrm{Na}$ inelastic & & & & & 3.99 & 3.55 & 2.26 & 2.25 & 3.73 & 3.77 & & & 6.18 & 6.60 \\
\hline${ }^{23} \mathrm{Na}$ capture & & & & & 1.07 & & & & & 1.09 & & & 1.81 & \\
\hline${ }^{23} \mathrm{Na}$ elastic-inelastic & & & & & -1.68 & -1.55 & -0.90 & -1.04 & & -1.59 & & -0.37 & -1.38 & \\
\hline${ }^{56} \mathrm{Fe}$ elastic & & & 0.58 & 0.47 & & & & & & & 0.37 & 0.72 & & \\
\hline TOTAL $^{\mathrm{c}}$ & 1.26 & 1.22 & 2.97 & 2.47 & 6.41 & 5.33 & 4.75 & 4.71 & 6.79 & 5.89 & 2.89 & 2.55 & 11.02 & 11.52 \\
\hline OVERALL $^{\mathrm{d}}$ & 1.318 & 1.28 & 3.06 & 2.61 & 6.69 & 5.58 & 4.94 & 4.92 & 7.04 & 6.16 & $\mathbf{3 . 0 3}$ & 2.68 & 11.50 & 12.10 \\
\hline
\end{tabular}

${ }^{a}$ Not computed.

${ }^{b}$ It refers to the cross correlations between elastic and inelastic cross sections.

${ }^{c}$ Total uncertainty from the reactions in the table. This can be computed from the individual values by adding the square of the positive values and subtracting the square of the negative values, then taking square root. Negative values are due to negative correlations or different signs of sensitivity coefficients for the correlated cross sections.

${ }^{d}$ Overall uncertainty (from all nuclide-reaction pairs).

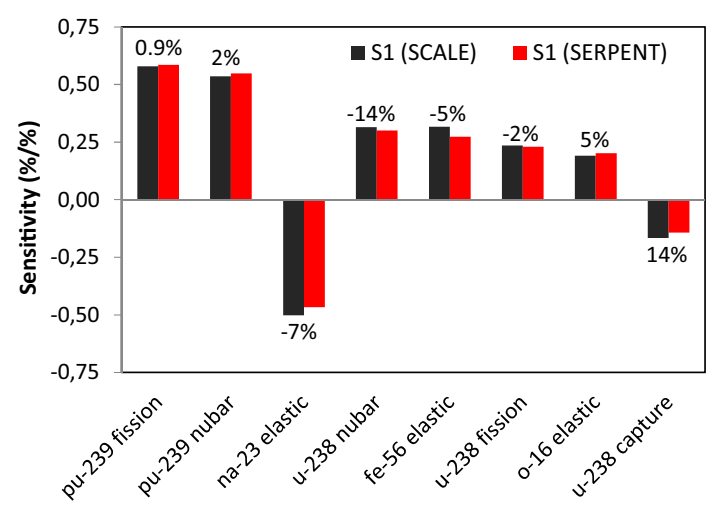

Figure 6. ISC of sodium void reactivity for scenario S1. Relative differences SERPENT-SCALE on the top of the bars.

in Table 4 are included in relative values in Table 3 for a better understanding.

It can be seen that for the sodium void uncertainties, not only fuel reactions are relevant (as for k-eff), but also the elastic and inelastic scattering of structural/coolant materials become important especially for $\mathrm{Na}$, stressing the fact that the dependencies of the reactivity coefficients on the nuclear data can be notably different from the dependencies of the multiplication constants of the nominal and perturbed states.

The smallest relative uncertainties $(\sim 3 \%)$ correspond to scenarios 1 and 5 where the sodium is voided from the plenum region. The leakage component is the dominant effect in those scenarios, showing that this component is
Table 4. Sodium-void worth and uncertainties (absolute values in pcm) using SCALE6.1 and SCALE6.2 (greyed) covariance data. Statistical uncertainties are included.

\begin{tabular}{|c|c|c|c|c|}
\hline \multirow[b]{2}{*}{ Scenario } & \multicolumn{2}{|c|}{ SCALE } & \multicolumn{2}{|c|}{ SERPENT } \\
\hline & $\begin{array}{l}\text { Void worth } \\
\text { (pcm) }\end{array}$ & $\begin{array}{c}\text { Uncert. } \\
(\mathrm{pcm})\end{array}$ & $\begin{array}{c}\text { Void worth } \\
\text { (pcm) }\end{array}$ & $\begin{array}{c}\text { Uncert. } \\
\text { (pcm) }\end{array}$ \\
\hline \multirow{2}{*}{ S1 } & \multirow{2}{*}{$-1413 \pm 13$} & $43 \pm 0.8$ & \multirow{2}{*}{$-1451 \pm 5$} & $38 \pm 0.7$ \\
\hline & & $58 \pm 0.9$ & & $50 \pm 0.7$ \\
\hline \multirow{2}{*}{$\mathrm{S} 2$} & \multirow{2}{*}{$439 \pm 13$} & $29 \pm 0.5$ & \multirow{2}{*}{$460 \pm 5$} & $26 \pm 0.5$ \\
\hline & & $33 \pm 0.7$ & & $29 \pm 0.5$ \\
\hline \multirow{2}{*}{$\mathrm{S}$} & \multirow{2}{*}{$326 \pm 12$} & $16 \pm 0.7$ & \multirow{2}{*}{$354 \pm 5$} & $17 \pm 0.7$ \\
\hline & & $19 \pm 1.0$ & & $20 \pm 0.7$ \\
\hline \multirow{2}{*}{ S } & \multirow{2}{*}{$219 \pm 12$} & $15 \pm 0.6$ & \multirow{2}{*}{$241 \pm 5$} & $15 \pm 0.6$ \\
\hline & & $17 \pm 0.9$ & & $17 \pm 0.6$ \\
\hline \multirow{2}{*}{ S5 } & \multirow{2}{*}{$-264 \pm 11$} & $8 \pm 0.8$ & \multirow{2}{*}{$-268 \pm 5$} & $7 \pm 1.0$ \\
\hline & & $10 \pm 1.3$ & & $11 \pm 0.9$ \\
\hline & \multirow{2}{*}{$203 \pm 12$} & $23 \pm 0.5$ & \multirow{2}{*}{$202 \pm 5$} & $24 \pm 0.6$ \\
\hline & & $24 \pm 0.8$ & & $24 \pm 0.7$ \\
\hline \multirow[t]{2}{*}{$\mathrm{All}^{a}$} & \multirow{2}{*}{$-536 \pm 12$} & $112 \pm 0.6$ & \multirow[t]{2}{*}{ - } & \multirow[t]{2}{*}{ 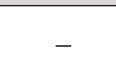 } \\
\hline & & $116 \pm 0.8$ & & \\
\hline
\end{tabular}

a $\mathrm{S} 1+\mathrm{S} 2+\mathrm{S} 3+\mathrm{S} 4+\mathrm{S} 5+\mathrm{S} 6$ voiding scenario. The obtained uncertainty is similar to other studies [10].

not very sensitive to nuclear data uncertainties. Scenarios 2,3 and 4 , corresponding to the voiding of different inner fuel zones and where the spectral component is the dominant effect, exhibit uncertainties up to $7 \%$. The largest uncertainty $(\sim 11 \%)$ occurs for scenario 6 .

Voiding all regions simultaneously leads to a negative sodium void coefficient and additivity of local reactivity 
effects is almost verified. But a large uncertainty is obtained since positive spatial correlations among the zones of the core increase the uncertainty in the sum of reactivities. Not considering correlations among the local uncertainties of the different zones would lead to underestimating the overall uncertainty during transient uncertainty propagation.

\section{Conclusions}

SCALE MG TSUNAMI-3D and GPT SERPENT2 modules were assessed for S/U analysis of an ASTRIDlike core. Very similar sensitivities were obtained for nominal k-eff while sensitivities for the sodium void reactivity effects exhibited larger differences (mainly with respect to elastic cross sections). The uncertainties in the sodium void reactivity depend on the voided core region, appearing slightly higher relative uncertainties when sodium is voided from the fuel regions. The breakdown contribution shows that ${ }^{238} \mathrm{U}$ inelastic and ${ }^{23} \mathrm{Na}$ elastic cross sections are the major contributors to sodium void uncertainties in plenum regions; ${ }^{23} \mathrm{Na}$ inelastic cross section is also a major contributor when voiding fuel regions. Those indications depend of course on the covariance library employed, and apply for both the SCALE6.1 and SCALE6.2 covariance libraries.

This work was conducted in the framework of the $7^{\text {th }}$ FP EU ESNII+ project (number 605172). Authors would like also to express their gratitude for the financial support of the $7^{\text {th }} \mathrm{FP}$ EU GENTLE project (number 323304) through the Student Research Experience Program.

\section{References}

[1] EC Community Research and Development Information Service - ESNII plus project. http:// cordis.europa.eu/project/rcn/110082_en. html

[2] P. Le Coz et al., Sodium-Cooled Fast Reactors : the ASTRID Plant Project, Proceedings of ICAPP 2011, Nice, France, May 2-5 (2011)

[3] MS. Chenaud et al., Status of the ASTRID core at the end of the pre-conceptual design phase 1, Nucl. Eng. Tech 45, 6 (2013)

[4] S. Bortot et al., European benchmark on the ASTRIDlike low-void-effect core characterization: neutronic parameters and safety coefficients, Proceedings of ICAPP 2011 Nice, France (2011)

[5] SCALE6.1, ORNL/TM-2005/39

[6] M. Aufiero et al., A collision history-based approach to sensitivity/perturbation calculations in the continuous energy Monte Carlo code SERPENT, Ann. Nucl. Energy 85 (2015)

[7] A.-L. Panadero, Static neutronic, thermal-hydraulic, and fuel performance analysis of the ASTRID Sodium-cooled Fast Reactor core (Lausanne, 2014)

[8] A. Martínez, Methodologies for $S / U$ analysis of the ASTRID-like SFR using SCALE system. Application to sodium voiding reactivity coefficients, MSc Thesis, UPM (2016)

[9] SERPENT -a Continuous-energy Monte Carlo Reactor Physics Burnup Calculation Code- website, http://montecarlo.vtt.fi/index.htm

[10] G. Aliberti et al., Nuclear data sensitivity, uncertainty and target accuracy assessment for future nuclear systems, ANE 33, 700-733 (2006) 